Pacific Journal of Mathematics

TOPOLOGIES ON THE RING OF INTEGERS OF A GLOBAL 


\title{
TOPOLOGIES ON THE RING OF INTEGERS OF A GLOBAL FIELD
}

\author{
JO-ANN COHEN
}

\begin{abstract}
A characterization of all nondiscrete, locally bounded ring topologies on the ring of integers of an algebraic number field and all those on the ring of integers of an algebraic function field for which the field of constants is bounded is given. As a consequence of these results we obtain Mahler's classic description of the seminorms on the ring of integers of an algebraic number field.
\end{abstract}

1. Introduction and basic definitions, Let $R$ be a ring and let $T$ be a ring topology on $R$, that is, $T$ is a topology on $R$ making $(x, y) \rightarrow x-y$ and $(x, y) \rightarrow x y$ continuous from $R \times R$ to $R$. A subset $A$ of $R$ is bounded for $T$ if given any neighborhood $U$ of zero, there is a neighborhood $V$ of zero such that $A V \subseteq U$ and $V A \subseteq U . \quad T$ is a locally bounded topology on $R$ if there exists a bounded neighborhood of zero for $T$.

We recall that a seminorm $\|\cdot\|$ on a ring $R$ is function from $R$ to the nonnegative reals satisfying $\|x\|=0$ if $x=0,\|x-y\| \leqq$ $\|x\|+\|y\|$ and $\|x y\| \leqq\|x\|\|y\|$ for all $x$ and $y$ in $R$. A seminorm on $R$ is a norm on $R$ if $\|x\|=0$ implies $x=0$.

If $\|\cdots\|$ is a seminorm on $R$, for each $\varepsilon>0$ define $B_{\varepsilon}=\{r \in R$ : $\|r\|<\varepsilon\}$. Then $\left\{B_{\varepsilon}: \varepsilon>0\right\}$ is a fundamental system of neighborhoods of zero for a locally bounded topology $T_{\|\cdot \cdot\|}$ on $R$. Two seminorms on $R$ are equivalent if they define the same topology.

An algebraic number field is a finite extension of the rational field $Q$. An algebraic function field is a finite extension of the field $F(x)$ of rational functions over the field $F$. A global field is either an algebraic number field or an algebraic function field.

Weber gave a complete description of the locally bounded topologies on the rational integers $Z$ [12] and Mahler characterized the normed topologies on the ring of integers of an algebraic number field [7]. A description of the locally bounded topologies on the ring of integers of $Q(\sqrt{-d})$ where $d$ is a positive, squarefree integer was obtained by Wieslaw [14]. In this paper we use Weber's description of the locally bounded topologies on global fields ([11, Theorem 3.3] and [13, Theorem 4.4]) to characterize all the locally bounded topologies on the ring of integers of an algebraic number field and also those on the integral closure of $F[x]$ in $K$ for which the subfield $F$ is bounded where $K$ is an algebraic function field. The results of Weber [12], Mahler [7], and Wieslaw [14] are a 
consequence of this characterization.

II. The ring of integers of a global field. If $K$ is an algebraic number field, let $R$ be the integral closure of the rational integers $Z$ in $K, P$ the set of nonzero prime ideals of $R$, and let $P_{\infty}$ be a set of archimedean absolute values on $K$ such that each archimedean absolute value on $K$ is equivalent to exactly one member of $P_{\infty}$. Then $R$ is a Dedekind domain properly contained in $K$ [5, Theorem 6.1, p. 23], $K$ is the quotient field of $R$ [10, Theorem I, p. 74] and $P_{\infty}$ is a finite set [5, Proposition 1.4, p. 81]. Denote $P \cup P_{\infty}$ by $P^{\prime}$.

If $K$ is a finite extension of $F(x)$, let $Z$ be $F[x], R$ the integral closure of $Z$ in $K, P$ the set of nonzero prime ideals of $R, v_{\infty}$ the valuation on $F(x)$ defined by, $v_{\infty}(f / g)=\operatorname{deg} g-\operatorname{deg} f,\left\{v_{1}, \cdots, v_{n}\right\}$ a complete set of extensions of $v_{\infty}$ to $K$ [1, Definition 3, p. 140; and Theorem 1, p. 143], and let $P_{\infty}=\left\{|\cdot|_{i}: i \in[1, n]\right\}$ where for each $i \in[1, n],|y|_{i}=2^{-v_{i}(y)}$ for all $y$ in $K$. Then $R$ is a Dedekind domain properly contained in $K$ and $K$ is the quotient field of $R$ [5, Theorem 6.1 , p. $23 ; 10$, Theorem I, p. 74]. As before, denote $P \cup P_{\infty}$ by $P^{\prime}$.

If $K$ is a global field, $R$ is called the ring of integers of $K$. Each $p \in P$ defines a $p$-adic valuation $v_{p}$ on $K$ (and hence on $R$ ) and furthermore, for each $a$ in $K \backslash\{0\}, v_{p}(a)=0$ for all but finitely many $p$ in $P[2, \mathrm{p} .25]$. Let $T_{p}$ denote the topology on $R$ associated with the valuation $v_{p}$. Then $\left\{p^{m}: m \geqq 0\right\}$ is a fundamental system of neighborhoods of zero for $T_{p}$. For each $p \in P$ and each $m \geqq 0$, let $T_{p}^{\prime} m$ be the topology on $R$ for which $\left\{p^{m}\right\}$ is a fundamental system of neighborhoods of zero. We note that $T_{p^{m}}^{\prime}$ is the topology on $R$ defined by the seminorm $\|\cdot \cdot\|_{p^{m}}$ where

$$
\|r\|_{p^{m}}=\left\{\begin{array}{ll}
0 & \text { if } r \in p^{m} \\
1 & \text { if } r \notin p^{m}
\end{array} .\right.
$$

Finally, for each $\mid \cdot \cdot \in P_{\infty}$, let $T_{\mid \cdot .}$ be the corresponding absolute value topology on $R$.

Henceforth, let $K$ be a global field and define $Z, R, P, P_{\infty}$ and $P^{\prime}$ as above. We denote topologies on $R$ by $T$ or $T^{\prime}$ and those on $K$ by $\hat{T}$.

LEMMA 1. Let $T$ be a ring topology on $R$ and let $|\cdot \cdot| \in P_{\infty}$. If $\{y \in R:|y|<M\}$ is a T-neighborhood of zero for some $M>0$, then $T \supseteqq T_{\mid . .1}$

Proof. Let $\varepsilon>0$. Then there exists a $c$ in $R$ such that $|c|>$ $M / \varepsilon$. Indeed, if $R$ is the ring of integers of an algebraic number 
field, let $c$ be a positive integer such that $|c|>M / \varepsilon$ and if $R$ is the ring of integers of an algebraic function field, let $c=x^{n}$ where $n$ is a positive integer such that $\left|x^{n}\right|>M / \varepsilon$. As $y \rightarrow y c$ is continuous at zero, there exists a $T$-neighborhood $U$ of zero such that $U c \leqq$ $\{y \in R:|y|<M\}$. Then for all $u$ in $U,|u||c|<M$ and hence $|u|<$ $\varepsilon$. Consequently, $\{y \in R:|y|<\varepsilon\}$ is a $T$-neighborhood of zero and so $T \supseteqq T_{1 . .1}$.

We recall that a subset $I$ of a field $F$ is an almost order of $F$ if (1) $0,1 \in I,(2)-I \subseteq I$, (3) $I I \subseteq I$, (4) there exists a nonzero element $h$ in $I$ such that $h(I+I) \subseteq I$, and (5) for each $x$ in $F^{*}$, there exist $y$ and $z$ in $I^{*}$ such that $x=y z^{-1}$. If $T$ is a nondiscrete, locally bounded ring topology on $F$, then there is an almost order $I$ of $F$ which is a $T$-bounded neighborhood of zero. Conversely, if $I$ is an almost order of $F$, then there exists a unique nondiscrete, locally bounded ring topology $T_{I}$ on $F$ for which $I$ is a bounded neighborhood of zero. Furthermore, $T_{I}$ is Hausdorff if and only if $I \neq F$ [6, Theorems 5 and 6].

We note also that if $U$ is a bounded neighborhood of zero for a topology $T$ on a field $F$, then $\{a U: a \neq 0\}$ is a fundamental system of neighborhoods of zero for $T$ [3, Exercise 20b, p. 120]. Consequently, if $U$ and $V$ are two $T$-bounded neighborhoods of zero, then there exist nonzero elements $a$ and $b$ such that $a U \subseteq V$ and $b V \cong U$.

Lemma 2. If $T$ is a nondiscrete, locally bounded topology on $R$, then there exists a T-bounded neighborhood $H$ of zero such that $H$ is a proper almost order of $K$. Furthermore, if a subset $A$ of $R$ is T-bounded, then $A$ is a $T_{H}$-bounded subset of $K$ as well.

Proof. Let $V$ be a $T$-bounded neighborhood of zero such that $1 \in V$ and $-V \subseteq V$. Define $H$ by, $H=\{r \in R: r V \subseteq V\} . \quad H$ clearly satisfies properties $1-3$ in the definition of an almost order. Since $V$ is a $T$-bounded neighborhood of zero, $H$ is a $T$-neighborhood of zero and as $1 \in V, H \subseteq V . H$ is therefore $T$-bounded as well. Consequently, $H+H$ is $T$-bounded and so as $T$ is nondiscrete, there exists a nonzero element $h$ in $H$ such that $h(H+H) \subseteq H . \quad H$ is a proper subset of $K$ since $H \subseteq R$. So it suffices to show that if $d$ is a nonzero element of $K$, then there exist nonzero elements $y$ and $z$ in $H$ such that $d=y z^{-1}$. Let $s$ and $t$ be nonzero elements of $R$ such that $d=s t^{-1}$. As the mappings $w \rightarrow s w$ and $w \rightarrow t w$ from $R$ to $R$ are continuous at zero, there exists a nonzero element $u$ in $H$ such that $s u$ and $t u$ are in $H$. Then $d=y z^{-1}$ where $y=s u$ and $z=t u$.

To prove the final assertion of the lemma, we note that if $A$ is $T$-bounded, then there exists a nonzero element $h$ in $H$ such that 
$h A \subseteq H$ as $T$ is nondiscrete. Hence $A$ is bounded for $T_{H}$ as well. For each nonempty subset $S$ of $P^{\prime}$, define $O(S)$ by,

$$
O(S)=\left\{y \in K: v_{p}(y) \geqq 0 \text { for all } p \in S \cap P\right.
$$

and

$$
\left.|y| \leqq 1 \text { for all }|\cdot \cdot| \in S \cap P_{\infty}\right\} .
$$

We note that $O\left(P_{\infty}\right) \cap Z$ is the set $\{0, \pm 1\}$ if $K$ is an algebraic number field. Therefore, if $T$ is a locally bounded topology on the ring of integers of an algebraic number field, then $O\left(P_{\infty}\right) \cap Z$ is bounded for $T$. We note further that if $K$ is an algebraic function field, then $O\left(P_{\infty}\right) \cap Z$ is the field $F$ of constants.

Weber proved that if $\hat{T}$ is any Hausdorff, nondiscrete, locally bounded topology on a global field $K$ for which $O\left(P_{\infty}\right) \cap Z$ is bounded, then there exists a nonempty, proper subset $S$ of $P^{\prime}$ such that $\hat{T}$ is the topology defined by the almost order $O(S)$ [11, Theorem 3.3 and 13, Theorem 4.4]. ( $S$ must be a proper subset of $P^{\prime}$. Indeed, if $K$ is an algebraic function field, then as $O\left(P^{\prime}\right)$ is contained in the set of elements of $K$ which are algebraic over $F$ [4, Corollary, p. 12], $O\left(P^{\prime}\right)$ fails to satisfy property 5 in the definition of an almost order. If $K$ is an algebraic number field, then $O\left(P^{\prime}\right)$ is finite [9, Theorem 33: 4, p. 69] and so once again, property 5 does not hold.) We use this result and the following lemma due to Seth Warner.

Lemma 3. If $A$ is a Hausdorff topological ring with identity 1 that has a bounded subfield $D$ containing 1 such that the left $D$ vector space $A$ is finite dimensional, then $A$ is discrete.

Proof. Let $n=\operatorname{dim}_{D} A$. First, $A$ is bounded, since if $\left\{e_{1}, e_{2}, \cdots\right.$, $e_{n}$ \} is a basis of $A$, then $A=D e_{1}+\cdots+D e_{n}$. We next show that any neighborhood $U$ of zero contains an open left ideal. Indeed, let $V$ be a neighborhood of zero such that $V+\cdots+V$ ( $n$ times) $\subseteq U$. As $A$ is bounded, there exists a neighborhood $W$ of zero such that $A W \subseteq V$. Let $\left\{a_{1}, \cdots, a_{m}\right\}$ be a maximal, linearly independent subset of $W$. Then $m \leqq n$ and $W \leqq D a_{1}+\cdots+D a_{m}$, for if $a \in W \backslash\left(D a_{1}+\cdots+D a_{m}\right)$ then $\left\{a_{1}, \cdots, a_{m}, a\right\}$ is a linearly independent subset of $W$. Thus $W \cong D a_{1}+\cdots+D a_{m} \subseteq A a_{1}+\cdots+$ $A a_{m} \leqq V+\cdots+V(m$ times $) \leqq U$ as $m \leqq n$. So $A a_{1}+\cdots+A a_{m}$ is the desired open left ideal contained in $U$.

As $A$ is finite dimensional over $D$ and therefore artinian, it has a minimal open ideal $J$ which is clearly not only minimal but the smallest. Consequently by the preceding, $J=(0)$ as $A$ is Hausdorff. So $A$ is discrete. 
THEOREM 1. Let $T$ be a nondiscrete, locally bounded topology on the ring $R$ of integers of a global field $K$ for which $O\left(P_{\infty}\right) \cap Z$ $i s$ bounded. Then there exists a proper subset $S$ of $P_{\infty}$, a subset $P_{1}$ of $P$ and nonnegative integers $n(p)$ for each $p \in P \backslash P_{1}$ such that

$$
T=\sup \left(\sup _{p \in P_{1}} T_{p}, \sup _{p \in P \backslash P_{1}} T_{p n \mid p\}}^{\prime}, \sup _{|\cdot| \in S} T_{|. . .|}\right) .
$$

In particular, if $K$ is an algebraic number field or if $K$ is a finite dimensional extension of the field $F_{q}(x)$ of rational functions over a finite 'field $F_{q}$ having $q$ elements, then every nondiscrete, locally bounded topology on $R$ can be described as above.

Proof. Let $H$ be a $T$-bounded neighborhood of zero such that $H$ is a proper almost order of $K$. Let $\hat{T}$ be the unique Hausdorff, nondiscrete, locally bounded topology on $K$ for which $H$ is a bounded neighborhood of zero. (We note that $\left.\widehat{T}\right|_{R} \supseteq T_{\text {.) }}$ By Lemma 2, $O\left(P_{\infty}\right) \cap Z$ is $\hat{T}$-bounded and hence by [11, Theorem $3.3 ; 13$, Theorem 4.4], there exists a proper subset $S^{\prime}$ of $P^{\prime}$ such that $\hat{T}$ is the topology associated with the almost order $O\left(S^{\prime}\right)$. So there exists a nonzero element a in $K$ such that $a O\left(S^{\prime}\right) \subseteq H \subseteq R$. If $O\left(S^{\prime}\right) \nsubseteq R$, let $y \in O\left(S^{\prime}\right) \backslash R$, let $p \in P$ be such that $v_{p}(y)<0$ and let $m>0$ be such that $v_{p}(a)+m v_{p}(y)<0$. Then $a y^{m} \in a O\left(S^{\prime}\right) \subseteq R$ but $v_{p}\left(a y^{m}\right)<$ 0. Contradiction! So $O\left(S^{\prime}\right) \subseteq R=O(P)$ and hence $O\left(P \cup S^{\prime}\right)=O\left(S^{\prime}\right)$. Therefore, $P \cup S^{\prime}$ is a proper subset of $P^{\prime}$ as $O\left(S^{\prime}\right)$ is an almost order of $K$ and so by replacing $S^{\prime}$ with $P \cup S^{\prime}$, we may assume that $S^{\prime}$ is a nonempty, proper subset of $P^{\prime}$ and $P \subseteq S^{\prime}$. Consequently, the set $S$ defined by, $S=S^{\prime} \cap P_{\infty}$, is a proper subset of $P_{\infty}$.

Let $|\cdot| \in S$. As $H$ is a $\hat{T}$-bounded neighborhood of zero, there exists a nonzero element $b$ in $K$ such that $b H \cong O\left(S^{\prime}\right) \subseteq O(\{|\cdot|\})$. So for each $h \in H,|h| \leqq 1 /|b|$. Consequently, $\{y \in R:|y|<2 /|b|\}$ is a $T$-neighborhood of zero and so by Lemma $1, T \supseteq T_{1 . .1}$.

For each $p \in P$, let $n(p)=\sup \left\{n \geqq 0: p^{n}\right.$ is a $T$-neighborhood of zero\}. Let $P_{1}=\{p \in P: n(p)=\infty\}$. We note that for all $p \in P \backslash P_{1}, p^{n}$ is $T$-open if and only if $0 \leqq n \leqq n(p)$ and for all $p \in P_{1}, p^{n}$ is $T$-open for $n \geqq 0$. Clearly, $T \supseteqq \sup \left(\sup _{p \in P_{1}} T_{p}, \sup _{p \in P \backslash P_{1}} T_{p n(p)}^{\prime}, \sup _{|. .| \in S} T_{\mid . .1}\right)$. So it suffices to prove that $T$ is weaker than the supremum topology.

Let $U$ be any $T$-closed neighborhood of zero. Then $U$ is a $\hat{T}$ neighborhood of zero as well. So there exists a nonzero element $a$ in $R$ such that $a O\left(S^{\prime}\right) \leqq U$. Let $p_{1}, \cdots, p_{m} \in P$ be such that $v_{p_{i}}(a)>$ 0 for $i \in[1, m]$ and $v_{p}(a)=0$ for $p \in P \backslash\left\{p_{i}: 1 \leqq i \leqq m\right\}$ [2, p. 25]. Consider the set $V \cap C_{1}$ where $V=\bigcap_{i=i}^{m} p_{i}^{v p_{i}(a)}$ and $C_{1}=\{y \in R:|y|<$ $|a|$ for all $|\cdot \cdot| \in S\}$. If $y \in V \cap C_{1}$, then $a^{-1} y \in O\left(S^{\prime}\right)$ and so $y \in$ $a O\left(S^{\prime}\right) \subseteq U$. Therefore, $V \cap C_{1} \subseteq U$. Let $\bar{V}$ be the T-closure of $V$ 
and define $W$ by, $W=\bar{V} \cap C_{2}$ where $C_{2}=\{y \in R:|y|<|a| / 2$ for all $|\cdot \cdot| \in S\}$.

We next show that $W \subseteq U$. Let $w \in W$ and let $W^{\prime}$ be any $T$ neighborhood of zero. As $T_{|\cdot .|} \subseteq T$ for all $|\cdot \cdot| \in S$, we may assume that $w^{\prime} \in C_{2}$ for all $w^{\prime} \in W^{\prime}$. Since $w \in \bar{V}$, there exists $w^{\prime} \in W^{\prime}$ such that $\quad w+w^{\prime} \in V$. Furthermore, $\quad\left|w+w^{\prime}\right| \leqq|w|+\left|w^{\prime}\right|<|a| / 2+$ $|a| / 2=|a|$ :for all $\mid \cdot \cdot \in S$ and so $w+w^{\prime} \in V \cap C_{1}$. Therefore, $w$ is in the $T$-closure of $V \cap C_{1}$. But $V \cap C_{1} \subseteq U$, a $T$-closed set. Hence $w \in U$ and consequently $W \subseteq U$.

To complete the proof of the theorem, it suffices to show that $W$ is open for the supremum topology. As $V$ is an ideal of $R, \bar{V}$ is also an ideal of $R$ [3, Proposition 5, p. 77] containing $V$. So $\bar{V}=$ $\bigcap_{i=1}^{m} p_{i}^{n_{i}}$ where $n_{i} \leqq v_{p_{i}}(a)$ for $i=1,2, \cdots, m$ [2, p. 26]. The canonical epimorphism $\phi$ from $R$ to $R / \bar{V}$ then defines a Hausdorff ring topology on $R / \bar{V}$ [3, Proposition 18, p. 25]. If $K$ is an algebraic number field, $R / \bar{V}$ is a finite ring [9, Theorem 33:2, p. 67] and the topology on $R / \bar{V}$ is therefore discrete. If $K$ is an algebraic function field, as $\phi$ is open and continuous, $\phi(F)$ is bounded. Furthermore, $R / \bar{V}$ is finite dimensional bover $\phi(F)$ [4, proof of Theorem, p. 23]. Therefore, by Lemma $3, R / \bar{V}$ is discrete. So in both cases, $\bar{V}$ is open for $T$. Thus for $1 \leqq i \leqq m, p_{i}^{n_{i}}$ is $T$-open and so $n_{i} \leqq n\left(p_{i}\right)$. Hence $W$ is open for the supremum topology.

Corollary 1. If $P_{\infty}$ has exactly one element, then the following statements are equivalent.

$1^{\circ}$. $T$ is a nondiscrete ring topology on $R$ for which the T-open ideals form a fundamental system of neighborhoods of zero.

$2^{\circ}$. $T$ is a nondiscrete ring topology on $R$ for which $R$ is bounded.

$3^{\circ}$. $T$ is a nondiscrete, locally bounded topology on $R$ for which $O\left(P_{\infty}\right) \cap Z$ is bounded.

Proof. Clearly $1^{\circ}$ implies $2^{\circ}$ and $2^{\circ}$ implies $3^{\circ}$. To prove that $3^{\circ}$ implies $1^{\circ}$ we need only notice that as the set $S$ defined in Theorem 1 is a proper subset of $P_{\infty}, S=\varnothing$.

CoRollary 2 [12, Theorem 1.5; 14, Theorem 1]. If $R$ is $Z$, $F_{q}[x]$, or the integral closure of $Z$ in $Q(\sqrt{-d})$ where $d$ is a positive, squarefree integer, then statements $1^{\circ}, 2^{\circ}$, and $3^{\circ}$ are equivalent.

Proof. Corollary 2 follows from Corollary 1 and the observation that $P_{\infty}$ has exactly one element. (The proof for $Q(\sqrt{-d})$ is the same as the proof of Corollary 3 to Theorem 3 of [8].) 
TheOREM 2. Let $K$ be a global field and let $R$ be the ring of integers of $K$. If $T$ is a nondiscrete ring topology on $R$, then the following statements are equivalent.

$1^{\circ}$. There exists a proper subset $S$ of $P_{\infty}$, a finite subset $P_{1}$ of $P$, a finite subset $P_{2}$ of $P \backslash P_{1}$ and positive integers $n(p)$ for $p \in P_{2}$ such that $T=\sup \left(\sup _{p \in P_{1}} T_{p}, \sup _{p \in P_{2}} T_{p}^{\prime n(p)}, \sup _{[. . \mid \in S} T_{|\cdot|)}\right)$.

$2^{\circ}$. $T$ is defined by a seminorm which is bounded on $O\left(P_{\infty}\right) \cap Z$.

$3^{\circ}$. $T$ is a locally bounded ring topology on $R$ for which $O\left(P_{\infty}\right) \cap Z$ is bounded and there exists a nonzero element $c$ in $R$ such that $c^{n} \rightarrow 0$ in $T$.

Proof. We first show that $1^{\circ}$ implies $2^{\circ}$. If $S \cup P_{1} \cup P_{2}=\varnothing$, then $T$ is defined by the seminorm $\|\cdot \cdot\|$ where $\|r\|=0$ for all $r$ in $R$. If $S \cup P_{1} \cup P_{2} \neq \varnothing$, then $T$ is defined by the seminorm

$$
\left.\sup \sup _{p \in P_{1}}|\cdots|_{p}, \sup _{p \in P_{2}}\|\cdots\|_{p^{n(p)}}, \sup _{|\cdot| \in S}|\cdots|\right)
$$

where for each $p \in P_{1},|r|_{p}=2^{-v_{p}(r)}$ for all $r$ in $R$.

Since $T$ is nondiscrete, if $T$ is defined by a seminorm $\|\cdot \cdot\|$, then there exists a nonzero element $c$ in $R$ such that $\|c\|<1$. Therefore, $c^{n} \rightarrow 0$ in $T$ and so $2^{\circ}$ implies $3^{\circ}$.

To prove that $3^{\circ}$ implies $1^{\circ}$, first notice that if $c^{m} \in p$ for some $m>0$, then $v_{p}(c)>0$. Define $n(p), P_{1}$ and $S$ as in Theorem 1 . Then $\{p \in P: n(p)>0\} \subseteq\left\{p \in P: v_{p}(c)>0\right\}$, a finite set [2, p. 25]. So $P_{1}$ is finite. Clearly $T=\sup \left(\sup _{p \in P_{1}} T_{p}, \sup _{p \in P_{2}} T_{p}^{\prime n(p)}, \sup _{|. .| \in S} T_{|. .|}\right)$ where $P_{2}=\left\{p \in P \backslash P_{1}: n(p)>0\right\}$.

CoRollary [7, p. 328]. If $\|\cdot\|$ is a seminorm on the ring of integers $R$ of a global $K$ for which $O\left(P_{\infty}\right) \cap Z$ is bounded and if $\|r\| \neq 0$ for some $r$ in $R$, then $\|\cdot\| \|$ is equivalent to the supremum of finitely many p-adic absolute values, finitely many seminorms $\|\cdot \cdot\|_{p^{n}}$ defined by the nonzero ideals $p^{n}$ of $R$, and finitely many absolute values from $P_{\infty}$.

\section{REFERENCES}

1. N. Bourbaki, Algèbre Commutative, Ch. 5 and 6, Hermann, Paris, 1964.

2. - Algèbre Commutative, Ch. 7, Hermann, Paris, 1965.

3. - Topologie Générale, Ch. 3 and 4, Hermann, Paris, 1960.

4. M. Deuring, Lectures on the Theory of Algebraic Functions of One Variable, SpringerVerlag, Berlin, 1973.

5. G. Janusz, Algebraic Number Fields, Academic Press, New York, 1973.

6. H-J. Kowalsky and H. Dürbaum, Arithmetische Kennzeichung von Körpertopologien,

J. Reine Angew. Math., (1953). 135-152.

7. K. Mahler, Über pseudobewertungen, III, Acta. Math., 67 (1936), 283-328.

8. E. Nichols and J. Cohen, Locally bounded topologies on global fields, Duke Math. J., 44 (1977), 853-862. 
9. O. T. O'Meara, Introduction to Quadratic Forms, Springer-Verlag, Berlin, 1971.

10. P. Ribenboim, Algebraic Numbers, Wiley-Interscience, New York, 1972.

11. H. Weber, Charakterisierung der lokalbeschränkten Ringtopologien auf globalen Körpern, Math. Ann., 239 (1979), 193-205.

12. —_, Ringtopologien auf $Z$ und Q, Math. Z., 155 (1977), 287-298.

13. - Topologische Charakterisierung globaler Korper und algebraischer Funktionenkörper in einer Variablen, Math. Z., 169 (1979), 167-177.

14. W. Wieslaw, Locally bounded topologies on some Dedekind rings, Archiv der Math., 33 (1979), 41-44.

Received November 14, 1979 and in revised form January 23, 1980.

North Carolina State University

RALEIGH, NC 27607 


\title{
PACIFIC JOURNAL OF MATHEMATICS
}

\section{EDITORS}

\author{
DoNALD BABBITT (Managing Editor) \\ University of California \\ Los Angeles, CA 90024 \\ Hugo RossI \\ University of Utah \\ Salt Lake City, UT 84112 \\ C. C. MOORE and ANDrew OGG \\ University of California \\ Berkeley, CA 94720
}

J. DugundJI

Department of Mathematics

University of Southern California

Los Angeles, CA 90007

R. FINN and J. MILGRAM

Stanford University

Stanford, CA 94305

\section{ASSOCIATE EDITORS}
R. Arens
E. F. BeCKenbaCH
B. H. NeumanN
F. WOLF
K. YoSHIDA

\section{SUPPORTING INSTITUTIONS}

UNIVERSITY OF ARIZONA

UNIVERSITY OF BRITISH COLUMBIA

CALIFORNIA INSTITUTE OF TECHNOLOGY

UNIVERSITY OF CALIFORNIA

MONTANA STATE UNIVERSITY

UNIVERSITY OF NEVADA, RENO

NEW MEXICO STATE UNIVERSITY

OREGON STATE UNIVERSITY

\author{
UNIVERSITY OF OREGON \\ UNIVERSITY OF SOUTHERN CALIFORNIA \\ STANFORD UNIVERSITY \\ UNIVERSITY OF HAWAII \\ UNIVERSITY OF TOKYO \\ UNIVERSITY OF UTAH \\ WASHINGTON STATE UNIVERSITY \\ UNIVERSITY OF WASHINGTON
}

The Supporting Institutions listed above contribute to the cost of publication of this Journal, but they are not owners or publishers and have no responsibility for its content or policies.

Mathematical papers intended for publication in the Pacific Journal of Mathematics should be in typed form or offset-reproduced, (not dittoed), double spaced with large margins. Please do not use built up fractions in the text of the manuscript. However, you may use them in the displayed equations. Underline Greek letters in red, German in green, and script in blue. The first paragraph or two must be capable of being used separately as a synopsis of the entire paper. Please propose a heading for the odd numbered pages of less than 35 characters. Manuscripts, in triplicate, may be sent to any one of the editors. Please classify according to the scheme of Math. Reviews, Index to Vol. 39. Supply name and address of author to whom proofs should be sent. All other communications should be addressed to the managing editor, or Elaine Barth, University of California, Los Angeles, California, 90024.

50 reprints to each author are provided free for each article, only if page charges have been substantially paid. Additional copies may be obtained at cost in multiples of 50 .

The Pacific Journal of Mathematics is issued monthly as of January 1966. Regular subscription rate: $\$ 102.00$ a year $(6$ Vols., 12 issues). Special rate: $\$ 51.00$ a year to individual members of supporting institutions.

Subscriptions, orders for numbers issued in the last three calendar years, and changes of address shoud be sent to Pacific Journal of Mathematics, P.O. Box 969, Carmel Valley, CA 93924, U.S.A Old back numbers obtainable from Kraus Periodicals Co., Route 100, Millwood, NY 10546.

\section{PUBLISHED BY PACIFIC JOURNAL OF MATHEMATICS, A NON-PROFIT CORPORATION}

Printed at Kokusai Bunken Insatsusha (International Academic Printing Co., Ltd.). 8-8, 3-chome, Takadanobaba, Shinjuku-ku, Tokyo 160, Japan. 


\section{Pacific Journal of Mathematics}

Vol. 93, No. $2 \quad$ April, 1981

Ilya Eugene Blum and Srinivasa Swaminathan, Continuous selections and

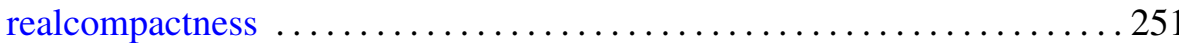

Lawrence James Brenton, Differentiably $k$-normal analytic spaces and extensions of holomorphic differential forms $\ldots \ldots \ldots \ldots \ldots \ldots \ldots 261$

Jo-Ann Deborah Cohen, Topologies on the ring of integers of a global field ................................................... 269

Robert Jay Daverman, Detecting the disjoint disks property $\ldots \ldots \ldots \ldots 277$

Edmund H. Feller, Rings where the annihilators of $\alpha$-critical modules are prime ideals ....................................... 299

Richard Elam Heisey and Henryk Torunczyk, On the topology of direct

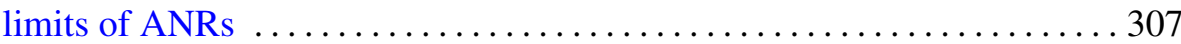

Gerald William Johnson and David Lee Skoug, Notes on the Feynman

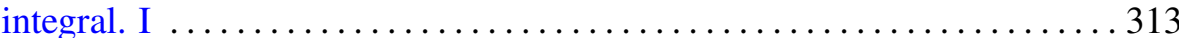

Michael S. Keane and Stuart Jay Sidney, Distinguishing a plane curve

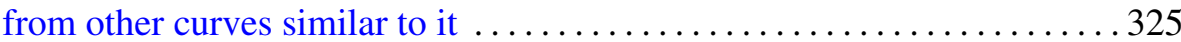

Leonid A. Luxemburg, On compact metric spaces with noncoinciding

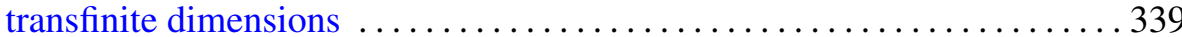

Chun Ming Ma, A uniqueness theorem for Navier-Stokes equations . . . . . . 387

Donald J. Newman and Theodore Joseph Rivlin, A characterization of the weights in a divided difference . . . . . . . . . . . . . . . . . . . 407

Marc Aristide Rieffel, $C^{*}$-algebras associated with irrational rotations $\ldots .415$

Kichi-Suke Saito, Invariant subspaces for finite maximal subdiagonal algebras

Frederic W. Shultz, Dual maps of Jordan homomorphisms and ${ }^{*}$-homomorphisms between $C^{*}$-algebras $\ldots \ldots \ldots \ldots$

Vsevolod Alekseevich Solonnikov, On the solvability of boundary and initial-boundary value problems for the Navier-Stokes system in domains with noncompact boundaries

Tavan Thomas Trent, New conditions for subnormality

L. E. Ward, Extending Whitney maps ..................

Leslie Wilson, Jets with regular zeros

Sergio Eduardo Zarantonello, The sheaf of $H^{p}$-functions in product 